\title{
Neuroma Prevention and Implantation Effects of NEUROCAP in Rat Sciatic Nerve Model
}

\author{
Steven L. Peterson, MD ${ }^{1}$ Harm de Vries, MSc ${ }^{2}$ Kami Collins, BA ${ }^{3} \quad$ Hilde Geraedts, PhD $^{2}$ \\ Michael J. Wheatley, MD ${ }^{4}$ \\ 1 Portland VA Medical Center, Portland, Oregon \\ 2 Polyganics, Groningen, The Netherlands \\ ${ }^{3}$ NAMSA, Brooklyn Park, Minnesota \\ ${ }^{4}$ Kaiser Permanente NW, Portland, Oregon

\begin{abstract}
Address for correspondence Hilde Geraedts, PhD, Polyganics, Groningen, Rozenburglaan 15A, 9727 DL, The Netherlands (e-mail: h.geraedts@polyganics.com).
\end{abstract}

J Reconstr Microsurg Open 2021;6:e1-e10.

\section{Abstract \\ Keywords \\ - symptomatic end- neuroma \\ - rat sciatic nerve model \\ - nerve-capping experimental design \\ - NEUROCAP}

Introduction Symptomatic neuroma with neuropathic pain can develop following peripheral nerve injury. Current interventions for symptomatic neuroma have unpredictable results. NEUROCAP (Polyganics, Groningen, The Netherlands) is a bioresorbable nerve capping device intended to protect a peripheral nerve end and separate the nerve from the surrounding environment, to prevent the recurrence of a symptomatic neuroma.

Materials and Methods This study aims to assess the implantation effects of the NEUROCAP device in a rat sciatic nerve model during 12 months ( \pm 2 days). Forty-one adult male Sprague-Dawley rats were used in this study. They were randomly divided into a capping or test group, or a noncapping or control group for different time points of survival (12 weeks, 6 months, and 12 months). The objective of this study was evaluated regarding procedural data, adverse events, clinical observations, and histopathology.

Results The overall general health of the animals was adequate throughout the study, with the exception of autotomy during the first 4 months of survival. Eight animals were euthanized early due to autotomy, excluded from the study and seven of them have been replaced. Autotomy was an expected outcome and a known limitation of the animal model, particularly as this was a full sciatic nerve transection model. Neuroma formation was observed in the control group while there was no neuroma formation present in the test group. The control group showed increased nerve outgrowth and more chaotic fascicles in comparison with the test group. The test group also had a higher percentage of myelinated fibers compared to the control group. These results indicate a preventive mode of action of the NEUROCAP with regard to neuroma formation after nerve transection in a rat sciatic nerve model.

Conclusion The results indicate that NEUROCAP is safe and effective in preventing the recurrence of neuroma formation and inhibiting nerve outgrowth. received

October 29, 2019

accepted after revision

November 1, 2020
DOI https://doi.org/

$10.1055 / \mathrm{s}-0040-1722201$. ISSN $2377-0813$.

\footnotetext{
(C) 2021. The Author(s).

This is an open access article published by Thieme under the terms of the Creative Commons Attribution-NonDerivative-NonCommercial-License, permitting copying and reproduction so long as the original work is given appropriate credit. Contents may not be used for commercial purposes, or adapted, remixed, transformed or built upon. (https://creativecommons.org/ licenses/by-nc-nd/4.0/)

Thieme Medical Publishers, Inc., 333 Seventh Avenue, 18th Floor, New York, NY 10001, USA
} 
A symptomatic neuroma may develop after a nerve dissection following any trauma to a peripheral nerve, whether accidental or planned (i.e., surgery). The incidence of symptomatic neuromas after peripheral nerve injury is estimated to be 3 to $5 \%$; however, certain surgeries (e.g., autograft procedures and amputation) may have up to a $30 \%$ incidence rate. ${ }^{1}$ Neuroma-induced neuropathic pain and morbidity can seriously affect the patient's daily life and socioeconomic functioning. $^{2}$

Multiple surgical procedures have been described to treat symptomatic end-neuromas, but none is considered gold standard for both treatment and prevention. ${ }^{3}$ The most common procedure is surgical removal of the end-neuroma and surrounding scar tissue, and placement of the proximal stump into an area subjected to minimal mechanical stimulation (e.g., soft tissue or bone). ${ }^{4}$ Guse and Moran reported recurrence rates up to $47 \%$ following neuroma treatment. ${ }^{5}$ In the late 1980s, degradable or absorbable materials became widely used to attain nerve regeneration. Currently, degradable polymers like polyglycolic acid, polylactic acid, and polyhydroxybutyrate are being studied and used as biodegradable and absorbable synthetic materials for nerve regeneration. ${ }^{6}$ Covering the nerve stump with a synthetic material that already has a proven track record in nerve repair bypasses possible biocompatibility issues with animal derived materials and led to the development of the NEUROCAP (Polyganics, Groningen, The Netherlands), a nerve capping device for the treatment of end-neuromas. ${ }^{7}$ Its composition is based on the same synthetic polymer conduits used in NEUROLAC nerve guide for the treatment of peripheral nerve lesions. NEUROCAP is a tubular device with one open end and one closed end. Dislocation of the nerve stump is prevented by suturing the nerve end into the cap. A hole at the sealed end of the tube allows easy fixation of the nerve stump with a suture to the surrounding tissue (-Fig. 1).

This study aims to assess the implantation effects of the NEUROCAP device in a rat sciatic nerve model after 12 weeks ( \pm 2 days), 6 months ( \pm 2 days), and 12 months ( \pm 2 days).

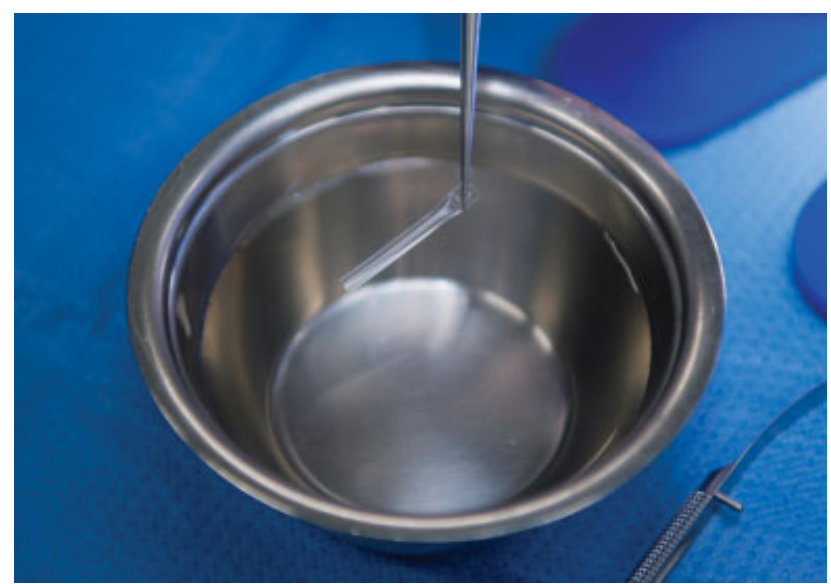

Fig. 1 The NEUROCAP device $(1.5 \mathrm{~mm})$.

\section{Materials and Methods}

\section{Study Population}

Forty-two adult male Sprague-Dawley rats weighing 243 to $501.3 \mathrm{~g}$ were used in this study. The primary end points of the study were the histology outcomes, and the use of all males ensured less variables without increasing group size. The primary objective of the study was to assess implantation effects of the test article, not gender differences. Therefore, the use of both male and female rats was not necessary to address our primary objective in this model.

The animals were randomly divided into a capping or test group and a noncapping or control group. The rats were assessed at 12 weeks ( \pm 2 days), 6 months ( \pm 2 days), and 12 months ( \pm 2 days) after treatment. Study group size was adjusted to accommodate for a larger group size at the primary endpoint at 6 months after implantation. At 12 weeks, each group included five animals. At 6 months, each group included eight animals. At 12 months, the nerve capping group included three animals and the control group included two animals. The remaining animals were lost to follow-up due to adverse events, as described in detail in the results section.

\section{Interventions}

The test article for this study was the NEUROCAP $1.5 \mathrm{~mm}$. The NEUROCAP is available in nine sizes (1.5-8.0 mm diameter), of which the smallest is used in this trial. This synthetically produced peripheral nerve capping device is composed of the bioresorbable copolyester poly(DL-lactide- $\varepsilon$-caprolactone). The NEUROCAP separates the nerve from the surrounding environment and protects the nerve end. The NEUROCAP elicits a minimal acute inflammatory reaction of the surrounding tissue, which is followed by gradual encapsulation of the cap by fibrous tissue. Degradation of the NEUROCAP occurs through hydrolysis leading to gradual reduction of molecular weight. The NEUROCAP retains sufficient mechanical properties to act as a barrier up to at least 10 weeks, where after rapid loss of mechanical strength and gradual mass loss occur. The final degradation products, lactic acid and hydroxy hexanoic acid, are resorbed, metabolized, and excreted by the body in approximately 16 months after implantation.

There was no control article used in this study. In the control group, the proximal nerve stump was left in situ.

\section{Study Design}

This study enrolled a total of 42 male Sprague-Dawley rats. Due to early deaths and exclusions, only 33 rats were evaluated throughout the study. Animals were assigned to each cohort according to the study design in -Table 1.

This Good Laboratory Practices (GLP) study was conducted in accordance with Food and Drug Administration Regulations on GLP for Nonclinical Laboratory Studies CFR, Title 21, Part 58 and applicable NAMSA standard operating procedures. 
Table 1 Study design

\begin{tabular}{|l|l|l|l|l|}
\hline Group & Article & $\mathbf{1 2} \mathbf{w k}$ & $\mathbf{6}$ mo & 12 mo \\
\hline Test & NEUROCAP & 5 & 8 & 3 \\
\hline Surgical control & None & 5 & 8 & 2 \\
\hline \multirow{2}{*}{ Early termination } & NEUROCAP & 0 & 0 & 1 \\
\cline { 2 - 5 } & None & 1 & 1 & 0 \\
\hline
\end{tabular}

\section{Surgery and Follow-Up}

Animals were sedated in accordance with testing facility standard procedures. The animals were intubated or masked and maintained under general anesthesia. The surgical sites were cleaned and shaved, and the animals transferred to the procedure room. Each rat was anesthetized with isoflurane 0.5 to $5 \%$ inhalant during induction, intraoperatively and prior to euthanasia as needed. Alternative or additional medications were given as prescribed by a test facility veterinarian.

Surgical procedures were performed under aseptic conditions. Incisions were made through the subcutaneous tissue on the lateral side of the right hind limb of each animal. The right sciatic nerve was exposed between the biceps femoris and the gluteal muscles. The sciatic nerve was transected with an Iris scissors at the trifurcation of the tibial, peroneal, and cutaneous branches.

In the NEUROCAP groups, the sciatic nerve was dissected and the nerve stump inserted approximately $5 \mathrm{~mm}$ into the device. The device was sutured to the device by means of two sutures (-Fig. 2).

In the control groups, the sciatic nerve was dissected, but the nerve stump remained in situ and the distal end was sutured to the surrounding muscle tissue. In both groups, a gap of at least $15 \mathrm{~mm}$ was maintained distal to the transection site to avoid spontaneous nerve regeneration.

The incisions were closed and the animals recovered from surgery.

Animals were observed daily during follow-up and received analgesics as necessary. Animals were anesthetized via isoflurane prior to euthanasia. After completion of the designated survival period and termination, animals were examined. Implant- and nonimplant-related alterations were collected for microscopic evaluation.

\section{Histopathology}

Samples were collected for histopathology from the test and control nerves along with contralateral nerves. Additional tissue surrounding the nerves was also collected to evaluate effects of the device on surrounding tissues. Samples were processed by Scientific Solutions, LLC. Stains for histopathology included hematoxylin and eosin (H\&E), Luxol Fast Blue, and Neurofilament 200.

Histopathology samples were sent to a veterinary pathologist for evaluation.

Histopathology slides were of longitudinal sections of each nerve. Evaluation of histopathological markers was based on ISO 10993-6:2016. When appropriate, histology

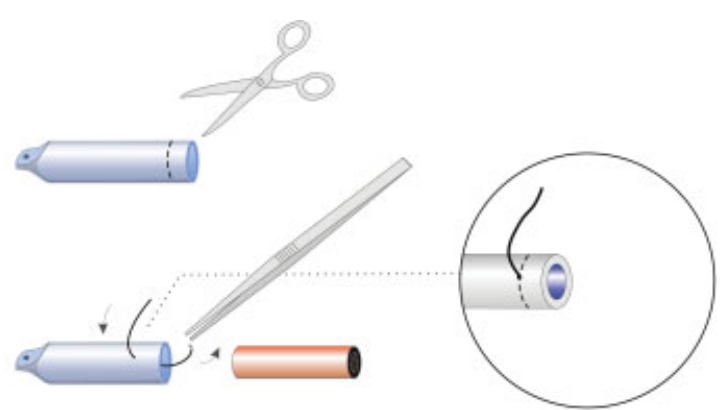

3

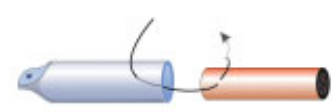

4

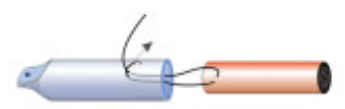

5

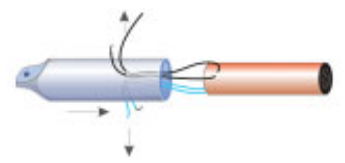

6

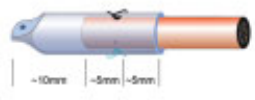

7

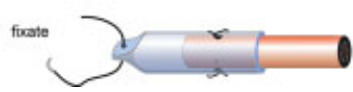

Fig. 2 Device sutures.

slides were evaluated for the percentage of unmyelinated fibers present by using the following scoring:

Evaluations were also made on the general orientation of the fibers, neuroma formation, nerve outgrowth, abnormal healing of the nerve, and any abnormal response in the surrounding nerve tissues. These outcome measures were either scored to be present or not present, or normal or abnormal where applicable, or were evaluated according to the grading scales in - Tables $\mathbf{2}$ and $\mathbf{3}$.

Nerves were evaluated in three zones. In the test article nerves, zone 1 was the tissue adjacent to the free end of the test article, zone 2 was nerve within the device at the cut and distal to the cut end (applicable if there is outgrowth of nerve tissue), and zone 3 was the nerve within the device proximal to the cut end. For the control article sites, zone 1 was the tissue adjacent to the cut end of the nerve, zone 2 was the nerve at the cut end and distal to the cut end (applicable if there is outgrowth of nerve tissue), and zone 3 was the nerve proximal to the cut end ( - Fig. 3 ).

After 12 weeks, 6 months, or 12 months, animals were humanely euthanized and tissues collected for histological evaluation. Slides were prepared by Scientific Solutions, LLC and stained with H\&E, LFB, and Neurofilament 200 (NF). The histological evaluation was conducted in accordance with the study protocol. Test and control nerves were stained with each of the three stains listed above (H\&E, LFB, and NF). Slides of contralateral nerves from test, and control groups were stained with LFB. 
Table 2 Grading for unmyelinated fibers

\begin{tabular}{|l|l|}
\hline Grade & Unmyelinated fibers observation \\
\hline 0 & No unmyelinated fibers present \\
\hline 1 & $1-25 \%$ unmyelinated fibers present \\
\hline 2 & $26-50 \%$ unmyelinated fibers present \\
\hline 3 & $51-75 \%$ unmyelinated fibers present \\
\hline 4 & $76-100 \%$ unmyelinated fibers present \\
\hline
\end{tabular}

\section{Results}

There were 42 animals enrolled in total throughout the first 12 weeks of the study duration.

Eight animals were euthanized early due to autotomy, and only seven animals were replaced due the timing of the early deaths and replacement surgeries. Two additional animals were added to the study in amendment 1 .

Ten animals were humanely euthanized at 12 weeks $( \pm 1$ day) for the first time point of the study. Sixteen animals were randomly selected and humanely euthanized at 6 months ( \pm 2 days) for the second time point of the study and five animals were humanely euthanized at 12 months ( \pm 2 days) for the third time point of the study. The animals were equally distributed in the control and test groups at all time points, with three animals in the test group and two animals in the control group at the 12-month time point.

\section{Clinical Observations}

Animals were observed daily from arrival and through the duration of the study by veterinarians, veterinary technicians, and animal care staff.

In the 12-week cohort, there were five observations of autotomy noted by a test facility veterinarian. There was no difference between the test and control groups with regards to autotomy numbers and severity. All autotomy instances at the
Table 3 Grading of severity for histopathologic markers

\begin{tabular}{|l|l|}
\hline Grade & Severity observation \\
\hline 0 & Absent \\
\hline 1 & Minimal \\
\hline 2 & Mild \\
\hline 3 & Moderate \\
\hline 4 & Marked \\
\hline
\end{tabular}

12-week termination were not resolved at termination. In the 6-month cohort, there were 13 observations of autotomy noted by a test facility veterinarian. All autotomy in this cohort was resolved after approximately 4 months of survival period. In the 12-month cohort, there were four observations of autotomy noted by a test facility veterinarian. In the NEUROCAP (test) group, and also in this cohort the autotomy was resolved after approximately 4 months of survival period.

Four of the rats in the 12-month cohort were obese, which is a common problem in aged male Sprague-Dawley rats. One sudden death noted in this cohort, which based upon postmortem investigation results, was most likely caused by secondary effects due to obesity.

There were no differences between the test and control groups with regards to clinical observations.

\section{Clinical Pathology}

Blood samples were collected prior to surgical procedures and termination for all animals. All values in both test and control groups were considered within the normal range except for some findings. Lymphocytosis was noted in one animal at termination and was likely a stress response prior to blood collection and not clinically significant. Hyperglycemia was noted in two animals at termination and was likely a postprandial response since rats were not fasted prior to blood collection.

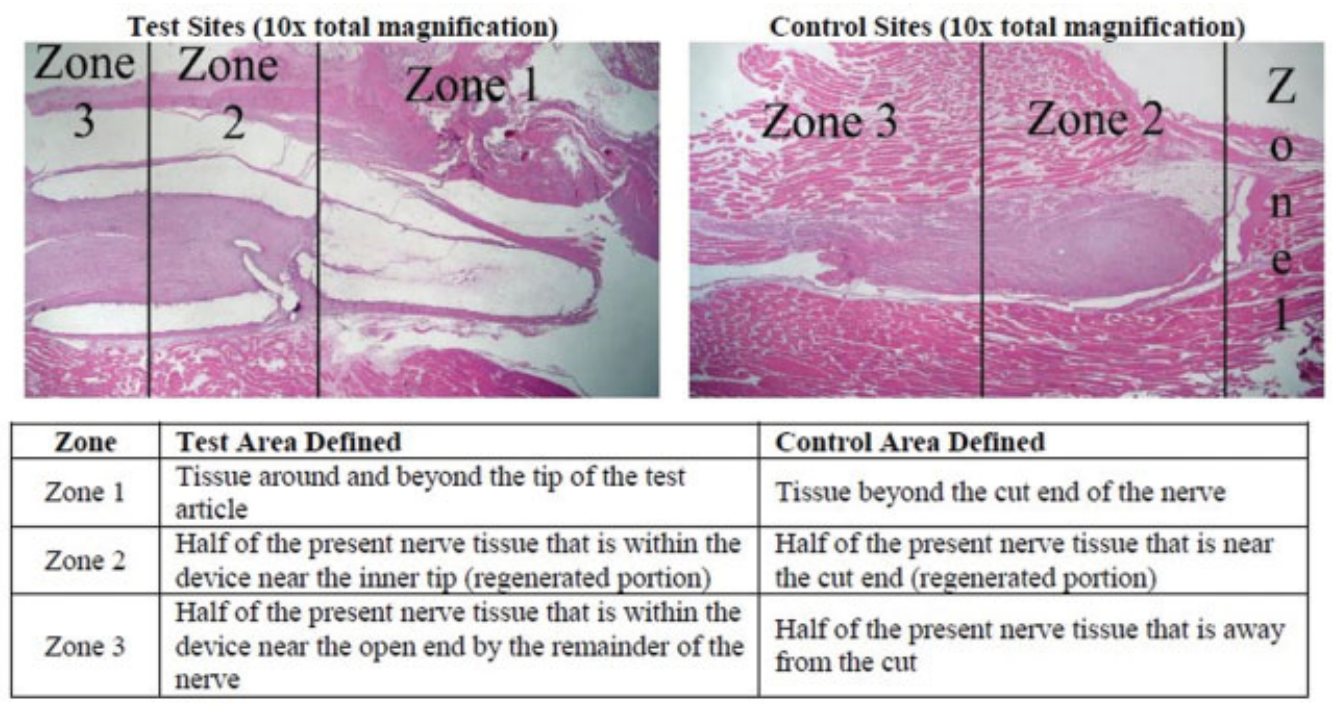

Fig. 3 Zone definitions. 
Table 4 Legend histopathology

\begin{tabular}{|l|l|}
\hline Z1 & Zone 1 \\
\hline Z2 & Zone 2 \\
\hline Z3 & Zone 3 \\
\hline AL & Axonal loss \\
\hline TA & Test article \\
\hline N & Nerve tissue \\
\hline NF & Neuroma formation \\
\hline S & Suture \\
\hline F & Fibrosis \\
\hline In & Inflammation \\
\hline ML & Myelination \\
\hline CF & Chaotic fascicles \\
\hline
\end{tabular}

Anemia and hypoalbuminemia noted in one animal at termination was likely related to its adverse event (heel pododermatitis).

There were no significant differences between the test and control groups with regards to clinical pathology outcomes.

\section{Adverse Events}

There were no adverse events related to the devices. All of the adverse events were related to the animal model and/or procedure. According to the physical examinations, the clinical pathology results, the daily observations, the adverse events, the surgical procedures, and the in-life assessment all animals enrolled in the study demonstrated adequate health to assess the objectives of the study.

\section{Histopathological Evaluation}

Histopathological outcomes were assessed for both groups at all time points. - Table 4 provides a legend for all histopathological pictures. - Tables 5-7 provide grading data of the outcomes for zone 2 for all time points. Independent sample $t$-tests were performed to assess differences between both groups at 12 weeks and 6 months after implantation.

Over time, the test nerves showed similar fibrosis with no neuroma formation in both zones 2 and 3 . The test nerves did show decreasing inflammation, chaotic fascicles, axonal loss, and increasing percent myelinated nerves in all zones over time. The control nerves showed similar fibrosis in both zones 2 and 3. There was increasing neuroma formation and chaotic fascicles in zone 2 and decreasing axonal loss for zones 2 and 3 for the control group over time.

Neuroma formation was not present in any of the test animals at any time point while two of eight control animals at 6 months, and two of two control animals at 12 months had neuroma formation present in zone 2 (-Fig. 4 ). - Figs. 5 and $\mathbf{6}$ provide representative images of neurofilament staining for zone 2 to evaluate axonal loss.

Over time, the test group had decreasing chaotic fascicles that had a lower average score than the control group, indicating that the nerves were becoming more organized in the test group while remaining less organized in the control group. The control group did have increased nerve outgrowth at all time points compared with the test group. In zones 2 and 3, the test group did have increased myelinated nerves over the course of 12 months compared with the control group, while the control group had similar myelination over the course of the 12 months. This indicates that long-term myelination of the nerves is not significantly affected by the test articled compared with the control. -Figs. 7 and $\mathbf{8}$ show representative images for chaotic fascicles and myelination in both test and control groups.

The test article showed resorption over time but was still present around the test nerve at the 12 month time point. The test group did have increased (minimal to mild) inflammation compared with the control group at both the 12week and 6-month time points, and this inflammation was associated with the test article material. The inflammation with the test article was an expected response to the implanted material. By the 12-month time point, there was no inflammation. - Figs. 9-14 provide representative images for inflammation markers, neuroma formation, and nerve outgrowth at all time points for both groups.

\section{Discussion}

In this study, the mode of action of a nerve capping device (NEUROCAP) for reduction of formation of neuroma was assessed in a rat sciatic nerve transection model. Results were compared with a control group in which the transected nerve end was left in situ without treatment. Results indicate that while in the test group, no neuroma formed during at the primary endpoint of 6 months after surgery as well as over the course of 12 months after surgery in the control group multiple neuroma formations were found during 12 months after surgery. Histopathologically, there were significant differences with regards to nerve outgrowth, chaotic fascicle formation, and percentage of myelinated fibers.

Neuroma associated neuropathic pain can be functionally debilitating and is notoriously difficult to manage. Serial interventions and a need for neuromodulating medications underscore the complexity of symptomatic neuroma management. Theoretically, intervening at the time of nerve injury to prevent the development of symptomatic neuromas can avoid much of the morbidity associated with peripheral nerve injuries.

Neuropathic pain is due to complex central and peripheral mechanisms that occur following nerve injury. ${ }^{8}$ Initially, the symptoms may be due to local inflammatory reactions that can irritate the nerve endings. Then a cascade of various cellular and humoral events occurs as macrophages and mast cells accumulate ate in the site of the lesion and inflammatory cytokines and factors are secreted to promote connective tissue scar formation. ${ }^{9}$ These inflammatory substances include histamine and serotonin which are responsible for pain production. ${ }^{10}$ Over time, C-fibers become sensitized 


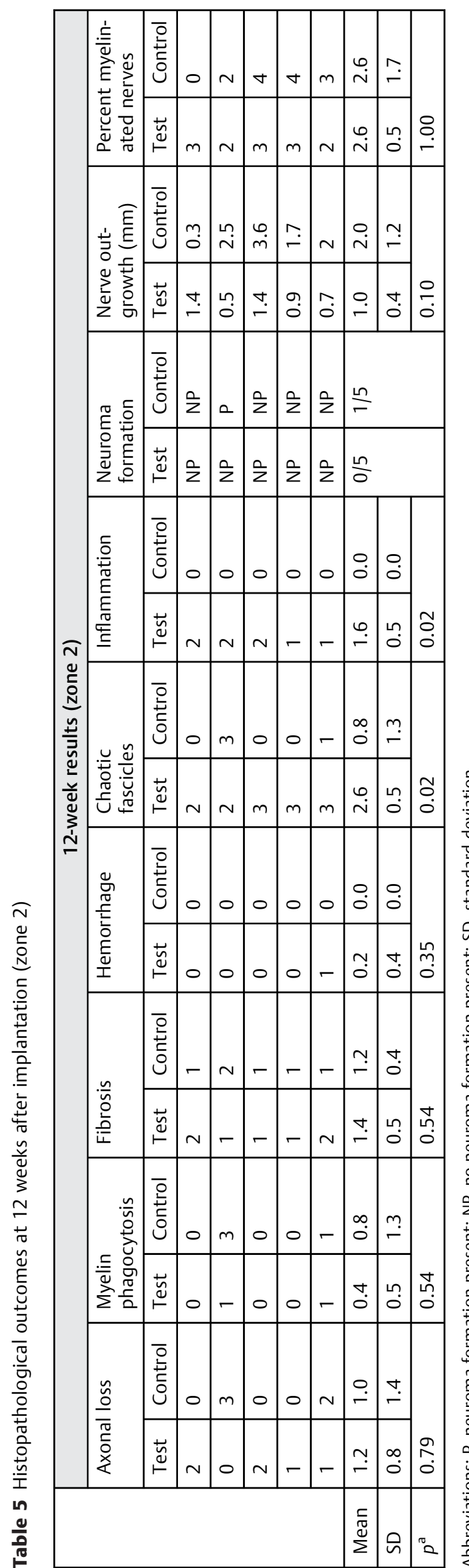

$\widehat{\mathrm{E}}$

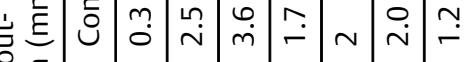

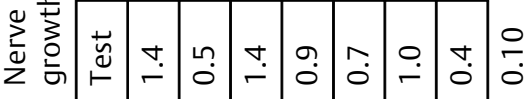

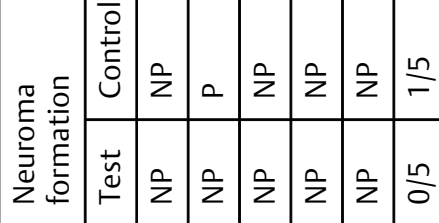

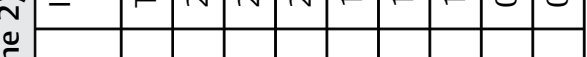

$\frac{n}{\frac{n}{5}}$

品

$\stackrel{\substack{1 \\ \sim}}{\sim}$

$\because \overline{\frac{2}{5}}$

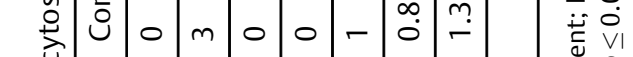

交薟

这

西

富

范

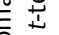

을 $\frac{\tilde{u}}{2}$

:

$\ddot{\mathrm{c}} \overrightarrow{\mathrm{C}}$

$\stackrel{0}{\frac{0}{0}}$

㐫

\begin{tabular}{|c|c|c|c|c|c|c|c|c|c|c|c|c|}
\hline \multirow{2}{*}{ 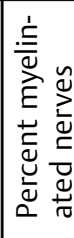 } & $\begin{array}{l}\overline{0} \\
\stackrel{0}{\underline{n}} \\
\overline{0}\end{array}$ & $\nabla$ & $\nabla$ & $m$ & $\nabla$ & $m$ & $\nabla$ & $m$ & $\sigma$ & $\stackrel{0}{m}$ & ㄴ?? & \\
\hline & $\stackrel{\vec{y}}{\ominus}$ & $\sigma$ & - & F & $m$ & $m$ & $m$ & $\nabla$ & $m$ & $\bar{m}$ & $\stackrel{\circ}{-}$ & $\stackrel{\sim}{\sim}$ \\
\hline 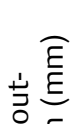 & $\begin{array}{l}\overline{0} \\
\stackrel{2}{\underline{L}} \\
\overline{0}\end{array}$ & $\stackrel{\infty}{\sim}$ & - & 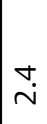 & 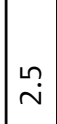 & $\stackrel{\stackrel{\sim}{\sigma}}{\sim}$ & $a$ & กै & 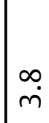 & $\stackrel{g}{m}$ & $\stackrel{\Delta}{i}$ & \\
\hline $\begin{array}{ll}2 \\
2 \\
2 \\
2\end{array}$ & 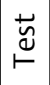 & $\sim$ & $\stackrel{\sim}{r}$ & $\stackrel{\varphi}{-}$ & $\stackrel{\sim}{\sim}$ & $\stackrel{\text { ㄴ? }}{r}$ & $\stackrel{m}{i}$ & 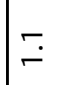 & $\widetilde{N}$ & $\stackrel{\text { ำ }}{\sim}$ & $\tilde{0}$ & $\begin{array}{l}0 \\
0 \\
0\end{array}$ \\
\hline \multirow{2}{*}{ 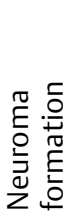 } & $\begin{array}{l}\overline{0} \\
\stackrel{0}{*} \\
\stackrel{0}{0} \\
\end{array}$ & $a$ & $\frac{n}{z}$ & $\frac{\hat{z}}{z}$ & $\frac{\hat{z}}{z}$ & $\frac{n}{z}$ & 0 & $\frac{a}{z}$ & $\frac{n}{z}$ & $\frac{\infty}{N}$ & & \\
\hline & $\begin{array}{l}\stackrel{\breve{n}}{\cong} \\
\vdash\end{array}$ & z & $\frac{\rho}{z}$ & $\frac{\rho}{z}$ & z & $\frac{n}{z}$ & $\frac{\hat{z}}{z}$ & $\frac{a}{z}$ & 之 & $\frac{\infty}{0}$ & & \\
\hline \multirow{2}{*}{ 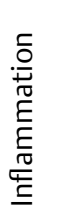 } & 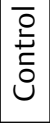 & 0 & 0 & 0 & 0 & 0 & 0 & 0 & 0 & O̊ & $\stackrel{\circ}{0}$ & \\
\hline & $\begin{array}{l}\overrightarrow{\tilde{s}} \\
\vdash\end{array}$ & $\sim$ & $\sim$ & - & - & - & - & - & - & $\stackrel{m}{r}$ & $\stackrel{n}{o}$ & $\stackrel{8}{0}$ \\
\hline \multirow{2}{*}{ 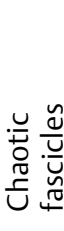 } & 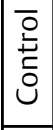 & $m$ & $\sim$ & $m$ & $m$ & $m$ & $m$ & $\sim$ & $m$ & $\stackrel{\infty}{\sim}$ & In & \\
\hline & $\stackrel{\overrightarrow{\tilde{g}}}{\mathscr{}}$ & $\sim$ & $\sim$ & $\sim$ & - & - & $\sim$ & - & $1-$ & $\stackrel{\text { In }}{\sim}$ & In & : \\
\hline \multirow{2}{*}{ 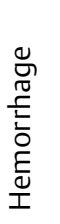 } & 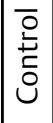 & 0 & 0 & 0 & 0 & 10 & 0 & 0 & 0 & $\stackrel{0}{0}$ & $\ddot{0}$ & \\
\hline & $\mid \begin{array}{l}\breve{g} \\
\vdash\end{array}$ & 0 & 0 & 0 & 0 & 10 & 0 & 0 & 0 & $\stackrel{0}{0}$ & 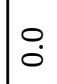 & $\stackrel{\circ}{\circ}$ \\
\hline \multirow{2}{*}{ 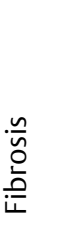 } & $\mid \begin{array}{l}\overline{0} \\
\stackrel{2}{ \pm} \\
0 \\
0\end{array}$ & - & $\sim$ & - & $\sim$ & - & $\sim$ & - & $1-$ & $\stackrel{+}{-}$ & In & \\
\hline & $\stackrel{\vec{n}}{\mathscr{a}}$ & $\sim$ & $\sim$ & - & - & - & - & - & - & $\stackrel{m}{\sim}$ & !n & 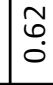 \\
\hline \multirow{2}{*}{ 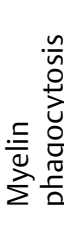 } & 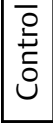 & - & 0 & 0 & 0 & 0 & 0 & 0 & 0 & : & $\stackrel{+}{0}$ & \\
\hline & 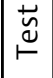 & 0 & 0 & - & 0 & 0 & 0 & 0 & - & $\stackrel{m}{0}^{m}$ & !n & 望 \\
\hline \multirow{2}{*}{ 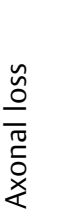 } & 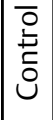 & 0 & 0 & - & - & $\sim$ & $\sim$ & $\sim$ & $\sim$ & $\stackrel{m}{r}$ & $\stackrel{9}{0}$ & \\
\hline & $\stackrel{\vec{n}}{\mathscr{a}}$ & 0 & $\sim$ & $v$ & $v$ & - & $m$ & $\sim$ & $\sim$ & $\stackrel{\infty}{\longrightarrow}$ & $?$ & 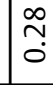 \\
\hline
\end{tabular}

$\widehat{E}$

定

离

(3)

$\stackrel{0}{\mathrm{E}} . \frac{5}{0}$

害它

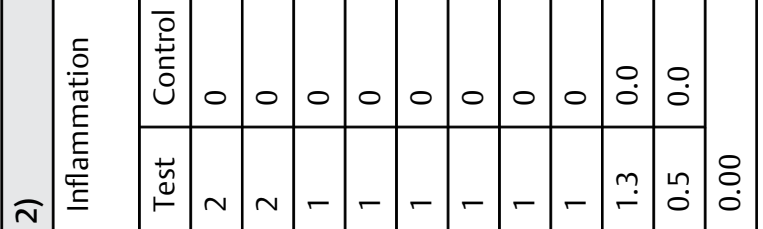

蒿

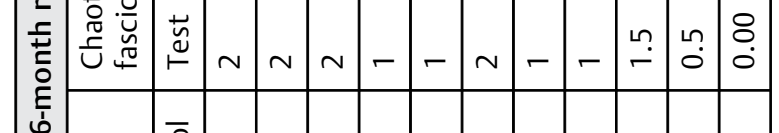

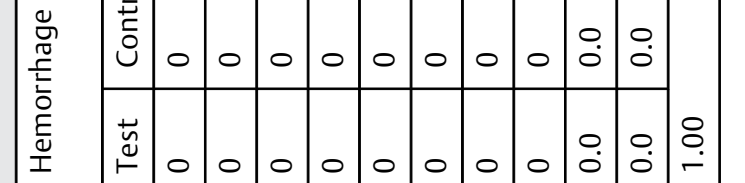

흐

흥 ฮั)

ก่

ज传

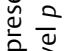
드 호 范 苞 $\frac{\tilde{u}}{0}$ $\ddot{\sim} \stackrel{0}{\sim}$ 芒蒙 음 这 


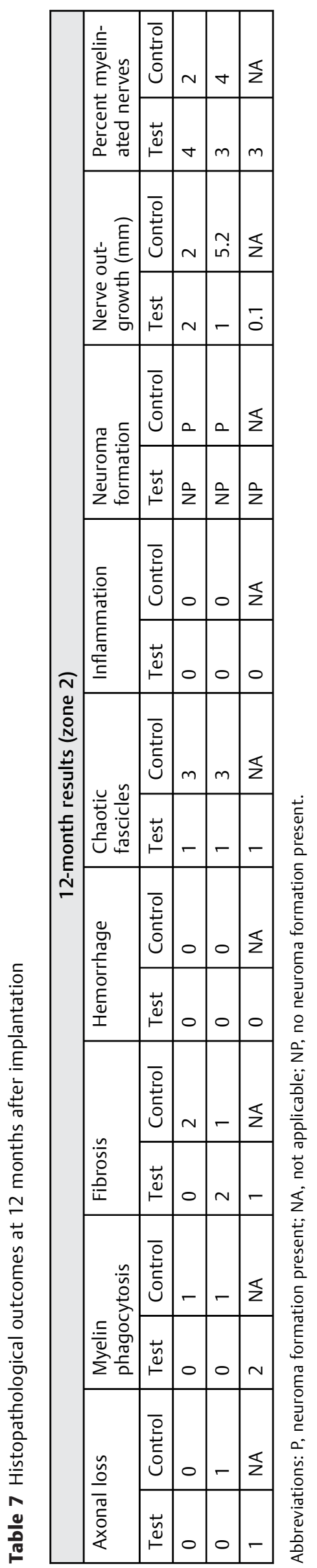

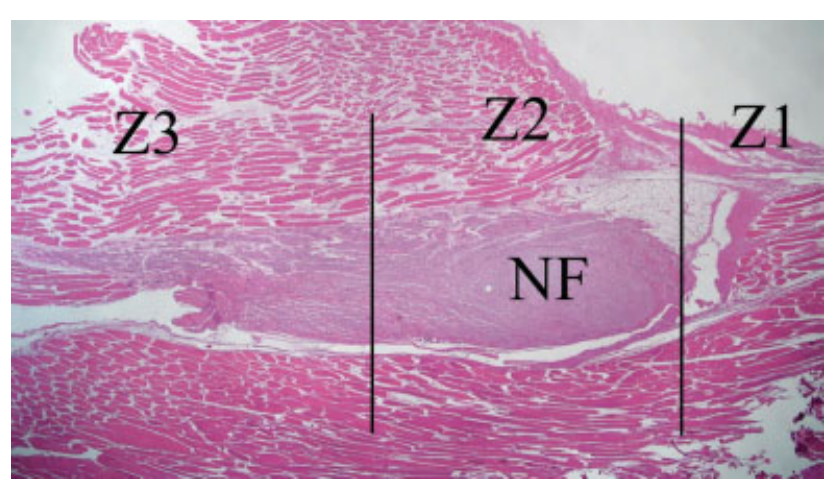

Fig. 4 Neuroma formation hematoxylin and eosin stain in control group animal at 12 weeks, $\times 10$ total magnification.

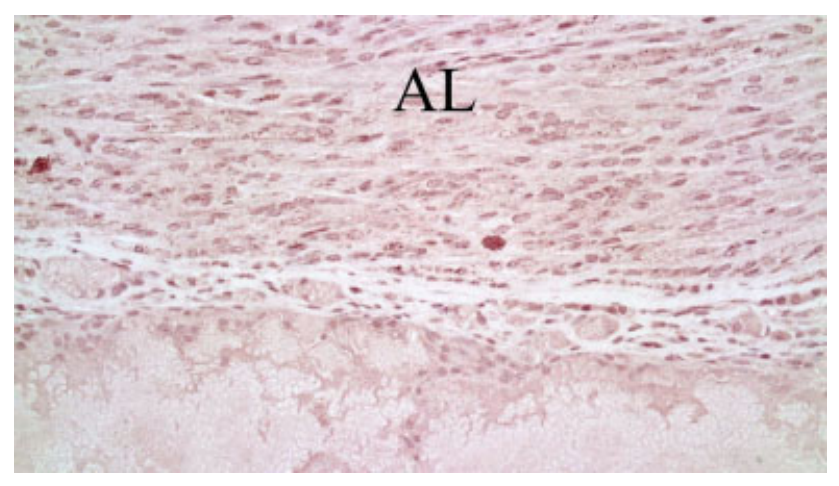

Fig. 5 Test group zone 2; 12 weeks after implantation; neurofilament stain; $\times 200$.

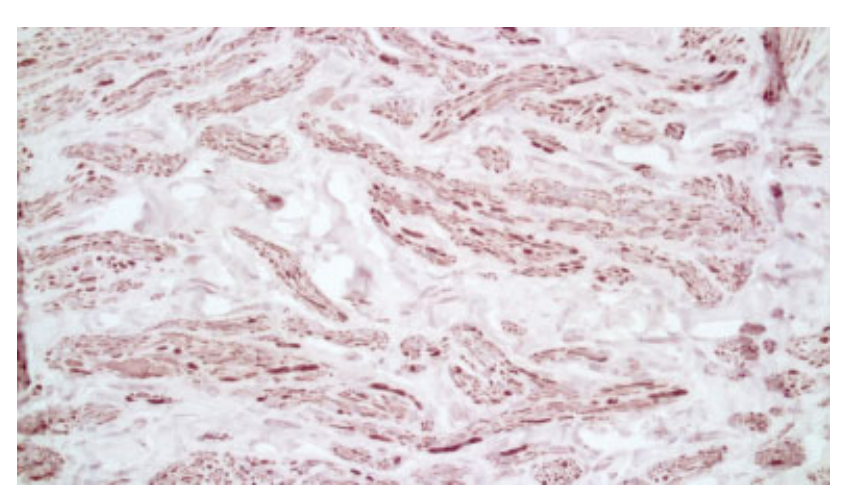

Fig. 6 Control group zone 2; 12 weeks after surgery; neurofilament stain; $\times 200$.

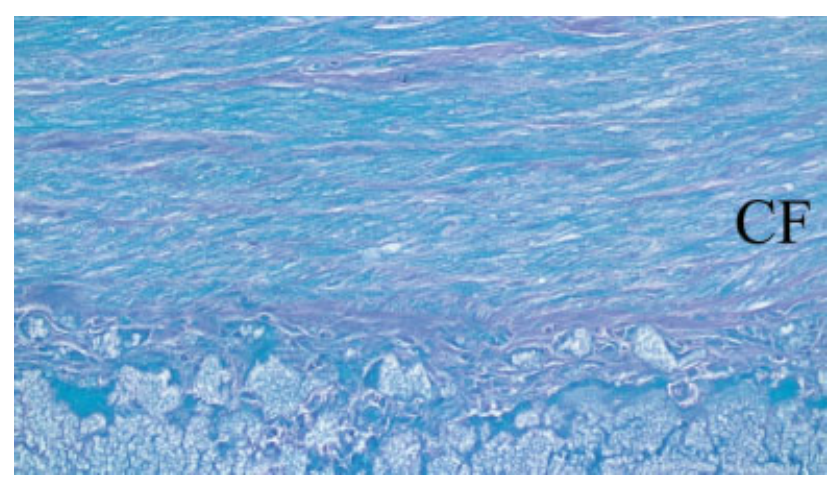

Fig. 7 Test group zone 2; 6 months after implantation; Luxol fast blue stain; $\times 200$. 


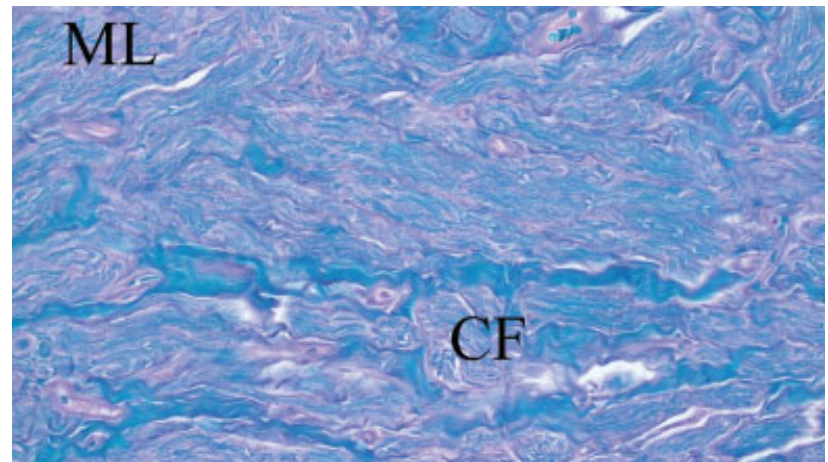

Fig. 8 Control group zone 2; 6 months after implantation; Luxol fast blue stain; $\times 200$.

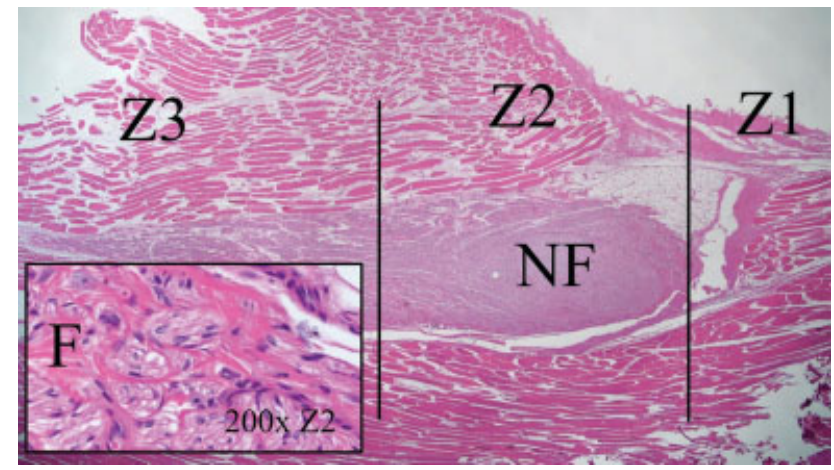

Fig. 10 Control group; 12 weeks after implantation; hematoxylin and eosin stain; $\times 10$ and $\times 200$.

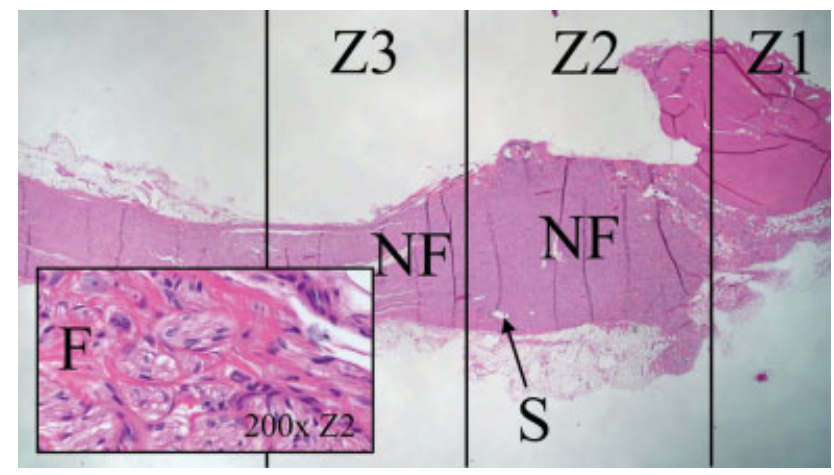

Fig. 12 Control group; 6 months after implantation; hematoxylin and eosin stain; $\times 10$ and $\times 200$.

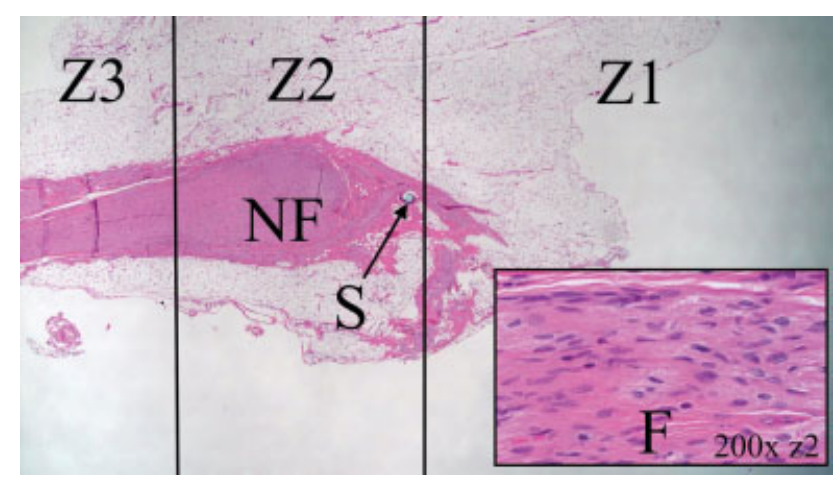

Fig. 14 Control group; 12 months after implantation; hematoxylin and eosin stain; $\times 10$ and $\times 200$.

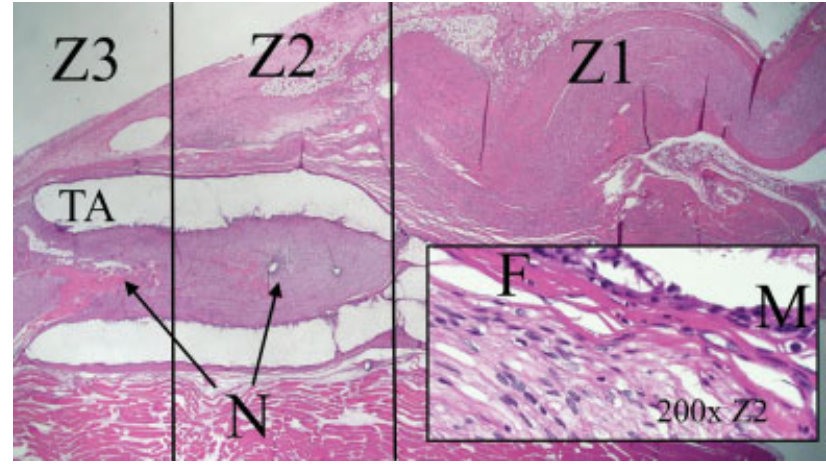

Fig. 9 Test group; 12 weeks after implantation; hematoxylin and eosin stain; $\times 10$ and $\times 200$.

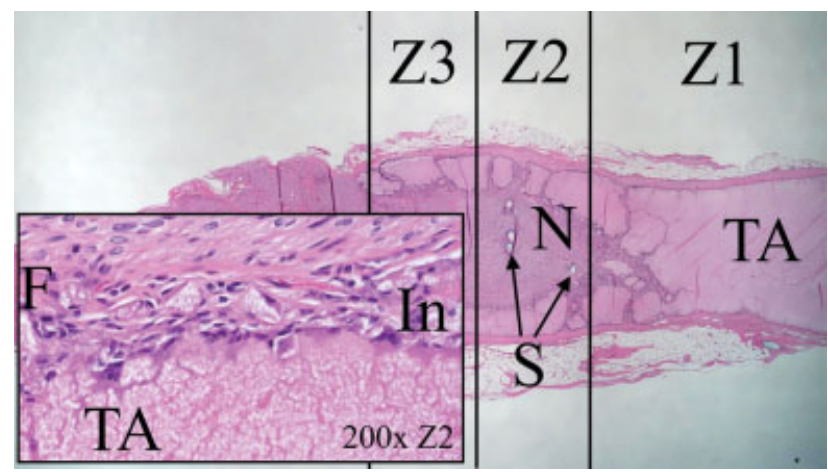

Fig. 11 Test group; 6 months after implantation; hematoxylin and eosin stain; $\times 10$ and $\times 200$.

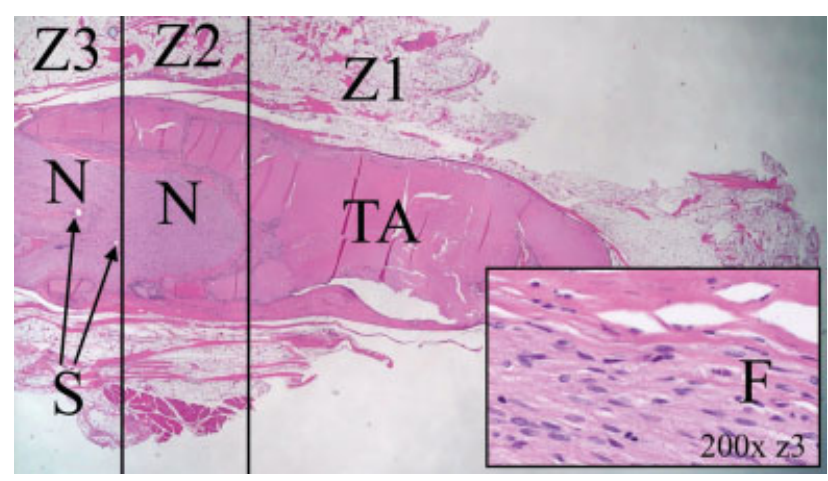

Fig. 13 Test group; 12 months after implantation; hematoxylin and eosin stain; $\times 10$ and $\times 200$.

pain generators. ${ }^{11}$ The injured nerve will attempt to regenerate, but in the absence of a distal target, axons that sprout can grow into the surrounding scar tissue, forming a neuroma. ${ }^{12}$ These results are illustrated by the occurrence of neuroma in the control groups in the current rat sciatic nerve transection model.

In clinical practice, a transacted nerve is often implanted into bone or a nearby muscle in an attempt to place a barrier between the anticipated neuroma and external stimuli. With this treatment, a different type of neuroma often develops that contains less connective tissue and is made up primarily of smaller and more painful sensory neurons. ${ }^{13}$ Nerve capping after stump excision has been well documented in the literature as an effective and widely used means of 
preventing symptomatic neuroma formation by modulating fiber outgrowth. ${ }^{6,14,15}$ The nerve cap forms a physical barrier that isolates the nerve from the surrounding inflammation and scar tissue. This barrier also serves to block the surrounding growth factors so as to change the regenerative response. ${ }^{16}$ Sunderland ${ }^{17}$ showed that the normal epineurium can prevent the lateral outgrowth of regenerating axons and that intact epineurium can control terminal neuroma formation by the isolation of neural tissues from the surrounding connective tissues. Epineural caps have been used in various studies, but the consistent problem of axonal escape remains. ${ }^{14-16}$ In history, silicone nerve capping devices have been used but have been linked to adverse events resulting from the use of silicone and requiring removal of the device. ${ }^{6}$ These experiences indicated a need for nonsilicone and bioresorbable nerve capping devices. Taneerananon et al have described the use of a NEUROLAC nerve guide, which is composed of the same bioresorbable material as NEUROCAP, formed as a cap around the severed nerve end by means as a capping device. ${ }^{15}$ The positive experiences in that study are emphasized by the current study results of a bioresorbable nerve capping device in a rat model, where a significantly lower number of neuroma occurrence was found in the NEUROCAP group when compared with the control group where the transected proximal nerve end was left in situ without treatment.

The rat sciatic nerve transection model is commonly used to study neuroma prevention and treatment. In this method, neuroma formation can be observed in rats as soon as 2 weeks after injury. ${ }^{18}$ In the current study, the macroscopic and histological effects of nerve capping after nerve transection in a rat sciatic model were studied at 12 weeks, 6 months, and 12 months after procedure. The benefits of the NEUROCAP became evident in the 6-month group and were confirmed in the 12-month group. The control group had increased nerve outgrowth in comparison with the test group as well as two sections with neuroma formation while there was no neuroma formation in any of the test sections. In addition, several of the control nerves had adhesions to the surrounding tissues, whereas adhesions were not noted in NEUROCAP test group. The gross necropsy findings regarding scarring in the groups correlated with the results from the histopathological evaluation where more fibrosis and inflammation was found in the surrounding tissues of the control group as compared with the test group. This scarring could be a source of traction neuritis that is thought to be a cause of neuropathic pain in symptomatic neuromas. At 6and 12-month time points, the test group had fewer chaotic fascicles compared with the control group, suggesting that the test nerves were becoming more organized and the control nerves were becoming less organized over time. Nerve outgrowth scores were significantly higher in the control group than in the test NEUROCAP group.

The study results showed that the NEUROCAP prevented both nerve tethering to surrounding tissues as well as chaotic nerve fascicle outgrowth. While this rat sciatic model does not allow for an analysis of neuropathic pain, both scarring and chaotic nerve outgrowth are thought to be the primary generators of the neuropathic pain seen in symptomatic neuroma, making it reasonable to anticipate that NEUROCAP placement following nerve injury or surgical intervention for symptomatic neuroma will decrease neuropathic pain. A prospective clinical trial is ongoing to test this specific hypothesis. Overall, the results in this rat sciatic nerve study confirm the findings of previous studies that indicate the preventive effect of a barrier around a severed nerve end to prevent formation of end-neuroma. ${ }^{15,16}$

\section{Strengths and Limitations}

The design of this study has several strengths. First, these are the first experimental trial results in an animal model addressing the mode of action and effectiveness of a nerve capping device for the treatment of nerve dissection. Second, the study includes histologic as well as macroscopic evaluation of results. Third, there is an extensive follow-up period of 12 months after implementation.

There are some limitations that influence the interpretation of the study. First, autotomy rates caused by the rat sciatic nerve model limit the available data, causing the 12month time point to be underpowered. However, the primary outcome was placed at 6 months after the procedure, which time point was adequately powered. Second, the clinically most important endpoint, whether the transected nerves are less symptomatic, could not be studied in this model. This is however further investigated in an ongoing prospective clinical trial to strengthen these data. Nevertheless, the absence of neuroma formation and adjacent tissue scarring and the formation of organized atrophied fibers at the nerve transection sites in the test group suggest that the NEUROCAP is modulating neuroma formation in ways that are likely to prove clinically beneficial.

\section{Conclusion}

This is a 12-month follow-up study on the efficacy and safety of a nerve capping device (NEUROCAP) in the prevention of neuroma in rats. The results show that NEUROCAP is able to prevent neuroma formation in a rat sciatic nerve model.

Conflict of Interest

H.G., M.J.W., K.C., H.D.V., and S.L.P. report personal fees from Polyganics B.V. during the conduct of the study and outside the submitted work.

\section{References}

1 Laing T, Siddiqui A, Sood M. The management of neuropathic pain from neuromas in the upper limb: surgical techniques and future directions. Plast Aesth Res 2015;2(04):165-170

2 Harden RN. Chronic neuropathic pain. Mechanisms, diagnosis, and treatment. Neurologist 2005;11(02):111-122

3 Vernadakis AJ, Koch H, Mackinnon SE. Management of neuromas. Clin Plast Surg 2003;30(02):247-268, vii

4 Balcin H, Erba P, Wettstein R, Schaefer DJ, Pierer G, Kalbermatten DF. A comparative study of two methods of surgical treatment for painful neuroma. J Bone Joint Surg Br 2009;91(06):803-808

5 Guse DM, Moran SL. Outcomes of the surgical treatment of peripheral neuromas of the hand and forearm: a 25-year 


\section{e10 Neuroma Prevention and Implantation Effects of NEUROCAP Geraedts et al.}

comparative outcome study. Ann Plast Surg 2013;71(06): 654-658

6 Biazar E, Khorasani MT, Montazeri N, et al. Types of neural guides and using nanotechnology for peripheral nerve reconstruction. Int J Nanomedicine 2010;5:839-852

7 Bertleff MJ, Meek MF, Nicolai JP. A prospective clinical evaluation of biodegradable neurolac nerve guides for sensory nerve repair in the hand. J Hand Surg Am 2005;30(03):513-518

8 Lewin-Kowalik J, Marcol W, Kotulska K, Mandera M, Klimczak A. Prevention and management of painful neuroma. Neurol Med Chir (Tokyo) 2006;46(02):62-67, discussion 67-68

9 Stoll G, Müller HW. Nerve injury, axonal degeneration and neural regeneration: basic insights. Brain Pathol 1999;9(02):313-325

10 Theodosiou M, Rush RA, Zhou XF, Hu D, Walker JS, Tracey DJ. Hyperalgesia due to nerve damage: role of nerve growth factor. Pain 1999;81(03):245-255

11 Nordin M, Nyström B, Wallin U, Hagbarth KE. Ectopic sensory discharges and paresthesiae in patients with disorders of peripheral nerves, dorsal roots and dorsal columns. Pain 1984;20(03):231-245
12 Maggi SP, Lowe JB III, Mackinnon SE. Pathophysiology of nerve injury. Clin Plast Surg 2003;30(02):109-126

13 Otfinowski J, Pawelec A, Kałuza J. Implantation of peripheral neural stump into muscle and its effect on the development of posttraumatic neuroma. Pol J Pathol 1994;45(03):195-202

14 Herndon JH. Neuromas. In: Green DP, Hotchkiss RN, eds. Operative Hand Surgery. Edinburgh: Churchill Livingstone; 1993: $1387-1400$

15 Taneerananon T, Hussain MA, Jhattu H, Li J, Klaassen M, Milovic V. Use of poly dl-lactide- $\varepsilon$-caprolactone (Neurolac) conduit for enveloping traumatic neuromas. Eur J Plast Surg 2013;36(10): 657-660

16 Barett S, Zgonis T. Nerve-Related Injuries and Treatments for the Lower Extremity. PA: Elsevier; 2016;(033)2:239-240

17 Sunderland S. The connective tissues of peripheral nerves. Brain 1965;88(04):841-854

18 Zeltser R, Beilin B, Zaslansky R, Seltzer Z. Comparison of autotomy behavior induced in rats by various clinically-used neurectomy methods. Pain 2000;89(01):19-24 